\title{
Liberalismus - Demokratie - Konservativismus: Moeller van den Bruck, das Begriffssystem eines Konservativen zu Beginn der Weimarer Republik
}

„Theorie und Praxis wirken immer aufeinander; aus den Werken kann man sehen, wie es die Menschen meinen, und aus den Meinungen voraussagen, was sie tun werden." (Goethe, Dichtung und Wahrheit 2, 7)

\section{Hinführung zum Thema}

November 1918: Der Krieg war verloren, der Kaiser geflohen und das Deutsche Reich nahezu über Nacht zu einer Republik geworden. Es war für viele Deutsche eine ungeliebte Republik, ein System, das ihnen von den westlichen Feinden aufgedrungen schien und das entsprechend in Verlängerung des verlorenen Krieges als feindliches System mit allen Mitteln bekämpft werden musste. Ermutigt wurden diese selbsternannten innenpolitischen Verteidiger des Vaterlandes durch den ,harten' Frieden von Versailles, die wirtschaftliche Krise, die dem Weltkrieg folgte, und durch die revolutionären Unsicherheiten, die das alte wilhelminische System im Rückblick als eine von Wohlstand und Sicherheit geprägte Zeit erscheinen ließen. Der Sprach- und Hilflosigkeit dieser konservativen Gruppierungen in den ersten Monaten nach dem Verlust des Reiches $^{1}$ folgte eine die ganze Weimarer Republik hindurch anhaltende Hetzkampagne gegen das Weimarer System, die schließlich in das dritte Reich Hitlers mündete. Viel gelesen wurde und großen Einfluss besonders bei den Jungkonservativen hatte dabei Moeller van den Brucks Buch „Das Dritte Reich“. 1923 zum ersten Mal erschienen, fand es zunächst noch wenig Leser, musste dann aber bereits 1926 zum zweiten Male aufgelegt werden und erfuhr während des Dritten Reiches noch zahlreiche weitere Auflagen. ${ }^{2}$ Die zweite Auflage hat der Autor bereits nicht mehr erlebt, da er im Herbst 1924 einen Nervenzusammenbruch erlitt und wegen seiner Zwangsneurosen in eine Nervenheilanstalt eingewiesen wurde. Moeller verübte am 30. Mai 1925 Selbstmord.

\footnotetext{
Von Emst Troeltsch einprăgsam als Traumland der Waffenstillstandsperiode bezeichnet, Troeltsch 1925, 69.

2 Dass sich die Nationalsozialisten spatestens im Jahre 1939 von Moeller distanzierten, soll hier nur als Ergănzung dienen. Die Distanzierung erfolgte u. a. in: Rodel 1939.
} 
Arthur Moeller van den Bruck gilt als konservativer Wegbereiter, sein Buch als ideologischer Vorläufer und vermeintlicher Namensgeber des dritten Reiches. Dennoch, und dies sei gleich zu Anfang erwähnt: Moeller van den Bruck war kein Nationalsozialist. Sein nationalchauvinistischer Ansatz, wobei die Betonung auf ,chauvinistisch' liegt, konnte mit dem ,ungebildeten' Führer der Nationalsozialistischen Partei, Adolf Hitler, nichts anfangen. Moeller van den Bruck gehörte allerdings zu denjenigen Konservativen, die mit einem überhöhten Nationalismus und einem radikalen Konservativismus den Weg für den Nationalsozialismus vorbereitet haben. Ich möchte sein Denken mit folgenden, schon jetzt zur Orientierung vorgestellten Thesen kennzeichnen:

(1) Das Diktum Kurt Sontheimers vom antidemokratischen Denken ${ }^{3}$ in der Weimarer Republik ist in Bezug auf Moeller van den Bruck wörtlich gar nicht und begrifflich nur dann gültig, wenn man es auf die heutige Begriffssetzung bezieht. Für Moellers Buch „Das Dritte Reich“ wäre wörtlich wie begrifflich die Kennzeichnung, antiliberales Denken' zutreffender. Moeller van den Bruck stellt deutlich die Dichotomie ,Liberalismus' versus ,Konservativismus' auf. Demokratie ist für ihn nur eine beliebig austauschbare und modifizierbare Staatsform, deren Akzeptabilität davon abhängt, ob eine bestimmte Form des Konservativismus in ihr realisiert ist.

(2) Moeller van den Bruck ist kein politischer Visionär, sondern ein Visionär des Unpolitischen. Er transponiert genuin politische Themen ins Unpolitische, das heißt konkret ins Charakterlich-Moralische und ins Geistig-Kulturelle. Gleichzeitig politisiert er das genuin Geistig-Moralische, und zwar in der Weise, dass er es metapolitisch, das heißt zur überparteilichen Größe und damit zum Maßstab sowohl der Politik als auch der Gesellschaft überhöht. Das Ergebnis dieser dritten Partei ${ }^{4}$ bzw. dieses dritten Standpunktes und schließlich auch des

3 Sontheimer 1994, 12f.: „Die innere Schwäche und das Sterben der Weimarer Demokratie sind unlöslich verknüpft mit der Wirksamkeit des antidemokratischen Denkens.“ Und S. 13: „Der Weimarer Republik fehlte ein Staatsbewusstsein, das sich an die demokratische Republik gebunden fuhlte und mit ihr in Einklang wußte." Auch Adorno nimmt das Problem des antidemokratischen Denkens als Grundfrage seiner Studie auf: „Wenn es ein potentiell faschistisches Individuum gibt, wie sieht es, genau betrachtet, aus? Wie kommt antidemokratisches Denken zustande?" Adorno 1996², 2.

+ Vgl. dazu: „Der Versuch, der in diesem Buche gemacht wurde, war nur von einem Standpunkte aus möglich, der keiner Partei verschrieben ist, vielmehr die ganze Spanne der Probleme einbezieht, die durch die Politik unserer Zeit gehen, von der außersten Linken bis zur äußersten Rechten: nur von einem dritten Standpunkte aus, der jeden anderen einschließt, den Parteideutsche haben konnen - von dem Standpunkte einer dritten Partei aus, die es bereits gibt. Nur ein solcher Versuch konnte sich, indem er die Parteien angriff, uber sie hinaus an die Nation wenden. Nur ein solcher Versuch konnte die deutsche Zerruttung und Zwieschaft aufzeigen, die aus langen Verhängnissen von den Parteien her, und durch sie, in unser politisches Leben getragen worden sind. Nur ein solcher Versuch konnte wieder die geistige Ebene politischer Anschauung feststellen, die von der Parteipolitik verlassen worden ist und die gleichwohl um der Nation willen gehalten, konservativ behauptet, revolutionär erstürmt werden muß." Moeller van den Bruck 1923, 6. 
von ihm angestrebten dritten Reiches ${ }^{5}$ ist der national-konservative Staat, der ohne Parteipolitik auskommt, und das heißt in letzter Konsequenz: unpolitisch ist. Moellers Ziel ist also die Entpolitisierung der deutschen Gesellschaft. ${ }^{6}$

(3) Entpolitisierung ist für Moeller van den Bruck die konservative Bewahrung aller guten, das heißt nationalen Werte und das Bekämpfen aller schlechten, das heißt liberalen Werte. Es geht ihm nicht um eine ausschließliche Erhaltung des Gewesenen, dies wäre für ihn reaktionär, sondern um radikale Durchsetzung des konservativen, das heißt nationalchauvinistischen Denkens. In diesem letzten Aspekt steckt das Revolutionäre, das revolutionär-konservative Element seiner Ideologie, die in letzter Konsequenz ideologisch den Weg für dynamische Neuordnungen mit konservativen Werthaltungen freimachen soll.

Durch die folgenden Ausführungen sollen sich zwei rote Fäden ziehen. Zum einen möchte ich eine Methode vorstellen, die zur semantischen Untersuchung historischer Texte dienen kann, und zum anderen möchte ich anhand dieser Methode vorführen, worin sich Moellers Opposition gegen die Republik äußert, warum sein Denken nicht antidemokratisch, sondern antiliberal war. Die hier vorgenommene Relativierung des Antidemokratischen soll den Blick frei machen für eine genauere Untersuchung aller Komponenten, die zum ideologischen und damit auch politischen Niedergang der ersten deutschen Republik gefürt haben. Ideologie soll im Folgenden im Sinne Adornos verstanden werden, nämlich:

\begin{abstract}
,an organization of opinions, attitudes and values - a way of thinking about man and society. We may speak of an individual's total ideology or of his ideology with respect to different areas of social life: politics, economics, religion, minority groups, and so forth. Ideologies have an existence independent of any single individual; and those which exist at a particular time are results both of historical processes and of contemporary social events.“"
\end{abstract}

Ideologien sind sprachliche Konstrukte, deren Träger das Ziel verfolgen, ihr Konstrukt von einer sprachlichen Existenzform in eine sachliche zu transportieren, d.h. die reale Welt in ihrem Sinne zu verändern. In linguistischer Perspektive sind sie also sprachlich-kommunikative Konstrukte, die mit dem Ziel propagiert werden, ihren sprachlich-kommunikativen Konstruktstatus als sach-

5 Vgl. dazu: „Das dritte Reich, wenn es je sein wird, schwebt nicht in Wohlgefallen hernieder. Das dritte Reich, das den Unfrieden endet, wird nicht in einem Frieden erstehen, der sich weltanschaulich verwirklicht. Das dritte Reich wird ein Reich der Zusammenfassung sein, die in den europäischen Erschütterungen uns politisch gelingen muß." Moeller van den Bruck 1923, 7; vgl. auch $40 \mathrm{f}$.

6 In diesem Zusammenhang sei der Hinweis auf Thomas Manns „Betrachtungen eines Unpolitischen" erlaubt. In den Betrachtungen finden sich deutliche inhaltliche Parallelen zu Moeller. Auch wenn man zwischen der ersten Auflage (1918) und der zweiten (1919) unterscheiden muss, so ist dieses Buch dennoch mit einem völlig anderen Geist geschrieben worden und diente intentional einem anderen Zweck. Der gravierendste Unterschied zwischen Moeller van den Bruck und Thomas Mann ist wohl der, dass sich Thomas Mann mit seinem Buch in keiner Weise aktiv in die Politik einbringen will, Moeller jedoch genau dieses zum Ziel hat.

7 Adorno $1964^{3}, 2$; in deutscher Übersetzung: Adorno $1996^{2}, 2$. 
adäquates, logisch begründetes oder aus anderen Gründen unbestreitbares Konzept von Realität oder gar als Realität überhaupt erscheinen zu lassen. Dieses Ziel ist maximal dann erreicht, wenn alle Gruppierungen der Öffentlichkeit dieser Meinung sind. Ein Konstrukt mit sprachlich-kommunikativer Existenzform ist also dann Realität, wenn es von allen Beteiligten als solche behandelt wird.

Auf dem Weg dazu muss eine Einbürgerung im Bewusstsein der Menschen erfolgen. Diese verläuft über die Schienen der Lexik und damit zusammenhängend der Bedeutungskonstitution. Ideologisch erfolgreich ist derjenige, der das sogenannte ,Besetzen' von Begriffen, das heißt die Bedeutungsgebung von Schlag-, Fahnen- oder Hochwertwörtern in optimaler Weise beherrscht. ${ }^{8}$

\section{Die Methode}

Die oben aufgeführten Thesen erfordern also zunächst einmal eine Untersuchung der von Moeller vorgenommenen Semantisierungen all derjenigen Ausdrücke, die nicht nur von Moeller van den Bruck in den Mittelpunkt der Diskussion gestellt wurden. Solche Untersuchungen können meines Erachtens am sinnvollsten mit einem lexikografischen Zugriff erfolgen, da nur so die schmale Gratwanderung zwischen Interpretation des Untersuchenden, Verifizierbarkeit durch den Leser der Untersuchung und Dokumentation auf begrenztem Raum möglich ist.

Unter , lexikografischem Zugriff ${ }^{\star}$ soll dabei eine Kombination folgender Arbeitsschritte verstanden werden:

(a) Der onomasiologische Arbeitsschritt; er besteht aus der Exzerption erstens aller lexikalischen Ausdrücke, die z.B. das Wortbildungsmorphem (-)demokrat(-) aufweisen, und zweitens all derjenigen lexikalischen Ausdrücke, mit denen Moeller nach dem beim ersten (onomasiologischen) Arbeitsschritt noch sehr vorläufigen Textverständnis auf dasjenige Bezug genommen hat, was ich als ,demokratisch' betrachte bzw. mir vorläufig als ,demokratisch' definiert habe. Der onomasiologische Arbeitsschritt beruht damit auf der Tatsache, dass man auf jeden Gegenstand, auf den man sprachlich Bezug nehmen will, in lexikalisch unterschiedlicher Weise Bezug nehmen kann. Das Ergebnis des onomasiologischen Arbeitsschrittes ist die Auffindung einer Reihe von Ausdrücken, die ich als ,Demokratiewörter' bezeichne, z.B. demokratisch, liberal, parlamentarisch, Republik.

(b) Die Exzerption der in a) gefundenen Demokratiewörter; sie soll hier als vorwiegend technischer Vorgang begriffen werden, ist aber in Wirklichkeit hochkomplex.

(c) Der semasiologische Arbeitsschritt; er mündet in der Erläuterung der Bedeutung jedes der im onomasiologischen Arbeitsschritt gewonnenen Wörter

8 Vgl. dazu die entsprechende Literatur, z.B. Liedtke 1991, Stotzel/Wengeler 1995, Hermanns 1995. 
bzw. in der Erläuterung mehrerer bis aller Bedeutungen dieser Wörter sowie (in letzterem Falle) in der Angabe inhaltlicher Beziehungen zwischen diesen Bedeutungen.

Der Vollzug der genannten drei Arbeitsschritte führt den Analysierenden von einem relativ vorläufigen, noch auf Vermutungen, Vorurteilen usw. beruhenden Einsichtsstand (eigentlich: Erwartungsstand) kontinuierlich zu einer Vertiefung der Einsicht und damit verbunden zur Möglichkeit ihrer wissenschaftlichen Begründung. Üblich ist der Fall, dass eine im onomasiologischen Arbeitsschritt gewonnene lexikalische Einheit sich beim Exzerpieren und erst recht während der semasiologischen Untersuchung als nicht zum Gegenstandsbereich gehörig oder als irrelevant erweist. Häufiger jedoch dürfte der Fall begegnen, dass man im semasiologischen Arbeitsschritt erkennt, einzelne oder mehrere gegenstandsrelevante Einheiten übersehen zu haben. Dies erfordert einen Arbeitsschritt (d) die Nachexzerption und daran anschließend wieder die Schritte b) und c).

Das Ergebnis historisch-semantischer Untersuchungen ist zu einem guten Teil Interpretation. Um so wichtiger ist es, dem Leser solcher Untersuchungen die erstellten Ergebnisse so durchschaubar zu machen, dass er relativ Gesichertes, nicht sinnvoll Bezweifelbares von dem auf begründeter Interpretation Beruhenden und von dem bloß Spekulativen unterscheiden kann. Die im wissenschaftlichen Text dargestellte Realität ist als größtmögliche Annäherung an die objekthistorische Realität zu konzipieren; dies setzt eine Anlage historischsemantischer Textsorten, z.B. von Wörterbuchartikeln, voraus, die den Leser in die Unterscheidung von Dichtung und Wahrheit einbeziehen.

Ein solcher Wortartikel hat dem angesprochenen idiolektalen Anliegen gemäß besondere Informationspositionen, die ich im Folgenden kurz vorstellen möchte:

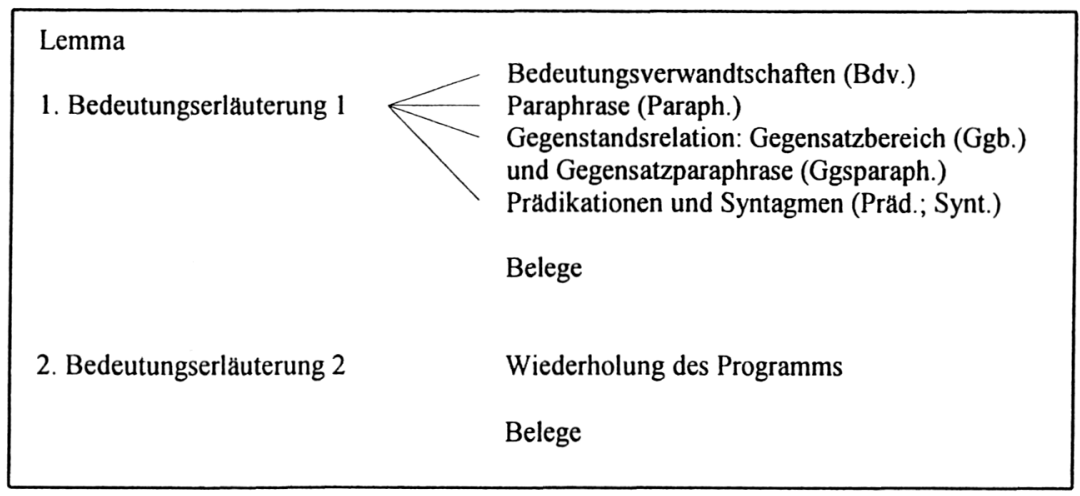

Abb. 1: Exemplarischer Aufbau eines Wortartikels

Die Bedeutungserläuterung ist die aus Belegbedeutungen abstraktiv gewonnene Formulierung einer Gebrauchsweise des Lemmazeichens. Bei einem Einzelautor, z.B. Moeller, ist dabei besonders darauf zu achten, dass genau dasjenige herausgestellt wird, was als individuelles Verständnis eines Ausdrucks (natürlich in 
seinem Kontext) zu verstehen ist. Die Wiedergabe der Bewertung und der einem Ausdruck innewohnenden bzw. vom Autor eingegebenen Deontik ${ }^{9}$ ist in diesem Zusammenhang nicht nur wünschenswert, sondern absolut notwendig.

In der Position ,Bedeutungsverwandtschaft' (Bdv.) stehen alle diejenigen lexikalischen Ausdrücke, die als partiell synonym mit dem Lemmazeichen, und zwar in der jeweils beschriebenen Bedeutung, interpretiert werden. Der Eintrag „Bdv.: liberal" s.v. demokratisch 3 bedeutet also, dass das Wort liberal als partiell synonym zu Bedeutung 3 von demokratisch zu sehen ist. Analog zur Betonung der Deontik in der Position ,Bedeutungserläuterung' ist auch hier darauf zu achten, dass sogenannte ,Wertsynonyme“ einbezogen werden. Das Ansatzkriterium ist dabei, ob Moeller das Lemmazeichen kontextuell durch das als bedeutungsverwandt angesetzte Wort substituieren könnte oder nicht. So ist es für ihn durchaus kein Problem, liberal an geeigneter Stelle durch verräterisch oder durch westlerisch zu ersetzen.

Unter der dritten Position, ,Paraphrase' (Paraph.), erscheint all dasjenige, womit der Autor im untersuchten Text ein Lemmazeichen (pro Bedeutung) paraphrasiert. Die Paraphrase das Aufgehen und die Hingabe zum Volksstaat wird dementsprechend als textinhaltlich weitgehend gleich mit demokratisch 2 interpretiert. Diese Informationsposition ist deswegen von besonderer Bedeutung, weil in ihr die autoreigenen Definitionen und definitionsähnlichen Erläuterungen (eben Paraphrasen) wiedergegeben werden können, ohne dass das Lemmazeichen dabei erscheint.

Als vierte Position wird der, Gegensatzbereich" aufgeführt. Unter der Sigle $G g b$. erscheinen alle diejenigen Ausdrücke, die zu einer bestimmten lexikalischen Einheit, z.B. zu demokratisch pro angesetzter Bedeutung als im Gegensatz stehend interpretiert werden. So ist z.B. liberal als Gegensatzwort zu konservativ auffassbar. Aufgrund der geringeren Häufigkeit wird an die Position Gegensatzbereich auch eine Gegensatzparaphrase angeschlossen, die ähnlich wie die Paraphrase, nur mit negativem Vorzeichen, dasjenige wiedergeben soll, was vom Autor als im Gegensatz zum Lemmazeichen stehend paraphrasiert wird.

Unter der Sigle Präd., ausformuliert Prädikationen, werden diejenigen Bezugsgrößen herausgestellt, denen das Lemmazeichen, also z.B. die Prädikation liberal, zugeordnet wird. Prädikationen charakterisieren Gegenstände, Sachverhalte oder Ereignisse hinsichtlich ihrer Qualität, Quantität, ihres Raumes oder Zeitpunktes näher bzw. setzen diese zu anderen Gegenständen und Sachverhalten in Beziehung. Prädikationen zum Substantiv Liberalismus können u.a. das Genitivattribut eines Westlertums oder das Adjektiv dumm sein. So ist es z.B. interpretationsrelevant, ob Liberalismus von Moeller nur politischen Bezugsgrößen wie ,Staat" oder auch charakterlichen Haltungen wie ,rechthaberisch" oder kognitiven Gegebenheiten wie ,Verstand' zugeordnet wird. Dies ist um so wichtiger, je mehr man über Bewertungen und Polemiken des Autors erfahren möchte. Der Kontextsensitivität wegen wird diese Position in der lexiko-

Vgl. dazu Hermanns 1994. 
grafischen Beschreibung am sinnvollsten mit der Syntagmenangabe in eins gesetzt und nur der Analyse wegen hier ausgesondert.

In der Position ,Syntagmen' (Synt.) stehen normalerweise nur diejenigen Ausdrücke, mit denen das Lemmazeichen syntagmatisch verbunden auftritt. Das Verb verführen z.B. verlangt zum einen ein Subjekt und zum anderen ein Akkusativobjekt. Daraus ergibt sich als Beispiel einer syntaktischen Grundform: jd./etw. (...) jn. (...) verführen. Die jeweiligen Klammern werden mit den im Text erscheinenden Ausdrücken gefüllt. Im oben genannten Beispiel lauten die entsprechenden Füllungen: Liberalismus und Sozialismus. Liberalismus ist Subjekt, Sozialismus Akkusativobjekt. Das Lemmazeichen erfährt auf diese Weise seine syntaktische Einordnung als Satzglied. Die korrekte syntagmatische Angabe lautet demnach: etw. (Liberalismus) etw. (Sozialismus) verführen. Da es aber nicht das Anliegen eines solchen Artikels ist, die syntaktischen Vorkommen eines Wortes zu dokumentieren, sondern das besondere Interesse darin besteht, die argumentativen Zusammenhänge zu beleuchten, in die ein bestimmtes Wort von seinem Autor gesetzt worden ist, dient die grammatische Einordnung vorwiegend der Strukturierung, der Verdichtung und der inhaltlichen Überschaubarmachung des zugrundeliegenden Materials. Die einzelne Syntagmenangabe folgt daher der Prämisse, den propositionalen Gehalt einer Aussage in kürzester Form zu kennzeichnen. Bei der Sytagmenangabe steht also der propositionale Gehalt einer Aussage im Vordergrund, der mit bzw. vermittels des angesetzten Lemmas oder Bezug nehmend auf das Lemma zum Ausdruck gebracht wird. Beim oben aufgeführten Beispiel steht folglich ausformuliert: Der Liberalismus hat den deutschen Sozialismus verführt. Oder: Der Konservativismus war die Voraussetzung, unter der wir den Krieg nur gewinnen konnten.

Wörterbuchartikel dieser Art haben vielfältige Funktionen. Da es das Anliegen der Autorenlexikografie ist, das Besondere und Eigene eines Autors speziell im inhaltlichen Bereich offen zu legen, müssen diejenigen Informationspositionen, die das spezifisch Idiolektale des zu behandelnden Textes aufnehmen können, engstens an den Quellentext rückgebunden sein. Dies ist mit den oben genannten Informationspositionen gewährleistet, da mit ihnen eben genau das Besondere und Eigene des Sprachgebrauchs, mehr noch die Assoziationen und Konnotationen, die gedanklichen Verknüpfungen und Unterscheidungen in der Vorstellungswelt des Autors, zum Ausdruck gebracht werden können. Besonders die Informationspositionen ,Paraphrase', ,Gegensatzbereich', ,Prädikationen“ und ,Syntagmen' wurden zu diesem Zwecke in die lexikografische Beschreibung eingefuihrt. Wie sich an anderer Stelle gezeigt hat ${ }^{10}$ und im Folgenden noch zu zeigen sein wird, kann dieser Artikelaufbau für die Interpretation von Texten eines einzelnen Autors, auch innerhalb eines historisch zurückliegenden Zeitraumes, sehr viel leisten. Er dient also sowohl der Bedeutungserschließung des einzelnen Lemmazeichens durch den Lexikografen wie der Erkenntnis der onomasiologischen Vernetzungen. Außerdem bietet ein solcher Artikelaufbau

10 Lobenstein-Reichmann 1998. 
sowohl für den Lexikografen als auch für den Leser die Möglichkeit der fortwährenden Rekontextualisierung, mit der auch immer eine Disziplinierung eigener Weltansichten verbunden ist. Je näher man, und damit sind wiederum sowohl der Lexikograf als auch der Leser gemeint, am Text bleiben kann, desto weniger läuft man Gefahr, den eigenen Horizont mit dem des geschichtlichen Autors zu verschmelzen. Es ist vielleicht an dieser Stelle nicht ganz fehl am Platz, darauf hinzuweisen, dass Horizontverschmelzungen nicht notwendigerweise über den Weg einer positiven Identifikation verlaufen müssen, sondern ebenso in einer vorgefassten negativen Bewertung des zu untersuchenden Gegenstandes begründet sein können. Gerade bei der Beschäftigung mit Texten, deren Inhalte nicht den eigenen gesellschaftlichen Wertvorstellungen entsprechen, ist eine Methode notwendig, die maximale Objektivität garantiert. Sie ist aber auch deswegen angeraten, weil man dabei von den Folgen und Konsequenzen solcher Texte, die der bearbeitende Lexikograf ja kennt, abstrahieren kann. Der allgemeine und zum Teil durchaus berechtigte Vorwurf, man könne die Weimarer Republik nicht nach ihrem Scheitern beurteilen, soll hier nur als eines von vielen Beispielen dienen. Ein weiteres Beispiel ist der zur festen Wendung gewordene Terminus vom , antidemokratischen Denken' in der Weimarer Republik.

Für den Leser bedeutet diese Vorgehensweise, wie oben bereits angedeutet wurde, dass auch er die Möglichkeit erhält, das Analysierte nachzuvollziehen, es zu verifizieren, gegebenenfalls aber auch, es zu falsifizieren. Darüber hinaus bekommt er einen schnellen Überblick über die Beleglage.

Die Schwierigkeit solcher Wörterbuchartikel besteht allerdings darin, dass der Bearbeiter einen Kompromiss finden muss zwischen einer möglichst vollständigen Dokumentation des vorgegebenen Materials einerseits und einer rezipientenfreundlichen Darbietung andererseits. Wählt der Bearbeiter nur wenige, besonders ,passende' Belege bzw. in diesem Fall Syntagmen aus, kann man ihm vorwerfen, er habe das Material seinen Ergebnissen angepasst. Die geforderte Nachprüfbarkeit durch den Leser wäre damit nicht mehr gewährleistet. Dokumentiert er jedoch das gesamte vorhandene Material, ist dieses nicht mehr wirklich durchdringbar. Im hier vorliegenden Argumentationszusammenhang muss ein Kompromiss eingegangen werden. Die Exzerption ist vollständig erfolgt und in die jeweiligen Artikel eingegangen. Das Material wird aber nur in einer pragmatischen Kurzform dokumentiert. Die Belegdarstellung wird vollständig weggelassen und in den Syntagmenangaben wurde stark gekürzt. Trotz weitgehender Kürzungen (z.B. im Bereich der Wiederholungen oder der Bezugsgrößen) soll versucht werden, dennoch den schmalen Grat zwischen einem Zuviel an Materialdarstellung und einem Zuwenig an Überprüfbarkeit zu gehen. Die Artikel sollen dabei innerhalb der Syntagmenangabe durch eine innere Strukturierung überschaubar gemacht werden. Dieser Kompromiss ist zugegebenermaßen unbefriedigend, lässt jedoch dem Leser einerseits die Möglichkeit offen, selbst zwischen einem kurzem Überblick und einer intensiveren Beschäftigung mit dem Material zu entscheiden. Der Volltext der Artikel soll an anderer Stelle einmal publiziert werden. 
Die onomasiologische Vernetzung, dasjenige also, was sich aus den Bedeutungsverwandtschaften ergibt, ist dann selbst wieder der Ausgangspunkt für weitergehende Analysen. Jedes als bedeutungsverwandt erkannte Wort wird (idealerweise) ebenfalls in einem Wortartikel bearbeitet, so dass auch von diesen Wörtern die onomasiologischen Vernetzungen eruiert werden können. Dieses aufwändige Verfahren dient dazu, den Weg von der Einzelbedeutung eines Einzellexems hin zum Begriff zu gehen. Der Schritt von der Größe ,Bedeutung' zu der Größe ,Begriff vollzieht sich wie folgt: Innerhalb eines Einzellexems wird immer von ,Bedeutungen' gesprochen (ein Wort hat, wie bereits normalsprachlich gesagt wird, ,Bedeutung'); die auf den Punkt gebrachte inhaltliche Gemeinsamkeit zweier oder mehrerer Einzelbedeutungen unterschiedlicher Lexeme wird hier als ,Begriff bezeichnet. Anders ausgedrückt: Alle über das Einzelwort hinausgehenden gemeinsamen Inhalte mehrerer Lemmazeichen werden letztlich auf einen vom Lexikografen erfassten Punkt, das heißt ,Begriff", gebracht. Begriffe sind mithin Zusammenfassungen des Analysierenden und somit interpretativ gewonnene kognitive Größen. Von ihnen wird allerdings angenommen, dass sie ein Analogon beim historischen Sprecher, in diesem Fall bei Moeller van den Bruck, haben."

Da bereits bei der Erstellung des semasiologischen Feldes zu jeder Einzelbedeutung bedeutungsverwandte Wörter angegeben wurden, erlauben die Wortartikel eine rasche Herausgliederung dieser Vernetzungen, also all derjenigen Ausdrücke, mit denen der historische Sprecher auf die pro Einzelbedeutung beschriebene Größe Bezug nehmen konnte. So besteht das Wortfeld von Demokratie aus bestimmten anderen Demokratieausdrücken wie z.B. Liberalismus, Parlamentarismus, wobei die beiden genannten als monosem angesetzt worden sind und daher keine Bedeutungszahl haben. Dasjenige also, was allen diesen Wortfeldgliedern inhaltlich gemeinsam ist bzw. als inhaltlich gemeinsam interpretiert wird, ist der Begriff. Der Begriff, auf den das Wortfeld von Demokratie gebracht wurde, ist ,Liberalismus'.

\section{Durchführung der lexikografischen Textanalyse}

Beim Betrachten des Artikels ,Demokratie' fällt auf, dass dieses Wort von Moeller van den Bruck ideologisch nur bedingt nutzbar gemacht wird. Im Gegensatz zu Konservativismus ist Demokratie kein Wort, mit dem er gesellschaftliche Veränderungen propagieren kann. Es ist nicht eindeutig genug und taugt nur bedingt dazu, absolute Wahrheiten zu verifizieren oder zu falsifizieren. Es ist nicht gruppenbildend. Demokratie ist zu Beginn des 20. Jahrhunderts, um es einmal etwas pointiert auszudrücken, kein Schibboleth mehr, an dem sich die Geister scheiden. ${ }^{12}$ Im Gegenteil, für Moeller ist es im Vergleich zu anderen

"Vgl. hierzu Lobenstein-Reichmann 1998, 23ff.; 2000, 193.

12 Vgl. dazu: Conze 1972, 898. 
politischen Schlagwörtern eher ein Schwamm, der alles aufsaugt und daher nichts mehr aussagt. ${ }^{13}$

Demokratie, die.

1. allgemein und generisch: >Volkssouveränität, Volksherrschaft (unabhängig von Staatsform, Ausprägung und Trägern der Staatsgewalt)<; mehrfach metonymisch: $>$ Nation, die eine demokratische Staatsform besitzt<; >politische Bewegung, die sich zum Ziel gesetzt hat, das Volk als Souverän eines Staates einzusetzen<; offen zu 2; 3. Paraph.: Anteilnahme des Volkes am Staate / an seinem Schicksal. - Präd. und Synt. (zum Subjekt): Demokratie kann Stoizismus bedeuten, Republikanertum, Unerbittlichkeit, Härte // Demokratie kann Liberalismus bedeuten, Geschrei in den Parlamenten und ein Sich-gehen-lassen in Wohlleben // Staat, in dem Demokratie und Konservativismus vereinbart sind; [...] (als Akk. Obj.): der liberale Mensch mißbraucht die Demokratie zu politischen Schutzformen // nicht die Staatsform macht eine Demokratie aus // andere Völker hatten die deutsche Demokratie verraten.

\section{Belege: $[\ldots]$}

2. >konservative, nationalistische, ständisch gegliederte Herrschaftsform mit einem starken, nahezu diktatorischen Staats- und Führerprinzip, das den angenommenen kollektiven Willen des Volkes bündelt und durchführt<; ütr.: >allgemeiner Wille des Volkes, jedoch nicht als einer Gruppe einzelner Individuen, sondern im Sinne eines Kollektivs, das nach einem als natürlich betrachteten, ständischen Prinzip geordnet ist<; Spezialisierung zu 1. - Bdv.: Stoizismus, Republikanertum, Unerbittlichkeit, Härte, Bindung, Volklichkeit. - Paraph.: Führerschaft und die Gefolgschaft / überhaupt eine Bindung, die selbst wieder auf Leitung, auf Gliederung, auf die Zelle zurückging "/ Volklichkeit, die Volksherrschaft nicht ist, sondern trägt. - Ggs.: Parlamentarismus; formale Demokratie. - Präd. und Synt. (zum Subjekt): Die deutsche Demokratie ist jetzt auf sich selbst angewiesen // wird national werden müssen // Demokratie ist der Ausdruck der Selbstachtung eines Volkes - oder sie ist nicht // die Demokratie wurde von den anderen Völkern verraten; (als Akk. Obj.): Nicht die Form des Staates, sondern der Geist seiner Bürger macht Demokratie aus; (als Prädikativum:) Wir waren eine Demokratie von Freien; (in präp. Angabe oder Ergänzung:) uns fehlt zur wirklichen Demokratie die demokratische Volksgesinnung, das Aufgehen und die Hingabe zum Volksstaat; (in präp. Attr.:) Wille zur Demokratie ist Wille zum politischen Selbstbewußtsein eines Volkes. und zu seiner nationalen Selbstbehauptung.

\section{Belege: $[\ldots]$}

3. >repräsentative Demokratie, pluralistische, parlamentarische, auf geheimem und gleichem Wahlrecht und durch eine Verfassung begründete Staatsform, die dem Willen einzelner Individuen nachgibt und nicht dem allgemeinen Wohle der Nation dient; alles gleichmachende, jeden Standes-, Leistungs- und Persönlichkeitsunterschied unterdrückende Herrschaftsform der Kompromisse, der Halbheiten, Hilflosigkeit und Tatenlosigkeit, der internationalen Völkerverständigung und des Kapitalismus $<$; spez.: $>$ Die Weimarer Demokratie, die Weimarer Republik<; Spezialisierung zu 1. - Bdv.:

13 Es folgen Artikelauszüge zu den Lemmata Demokratie bzw. demokratisch. 
Demagogie, Liberalismus, Parlamentarismus, Westlertum, Gemeinheit, Knotentum, Schiebertum, Halbintellektualismus. - Paraph.: die mechanische buchstabenmäßige, persönlich unschöpferische Durchführung eines Parlamentarismus /" liberales Chamäleon // Moloch, der Massen und Klassen und Stände und alle Unterschiede des Menschlichen frißt // Leviathan in seiner schillernden Ungeheuerlichkeit /I Bevormundung des Volkes, die Mundtotmachung ist // Staatsform, bei der jede Partei auf ihre Kosten kommt; - Ggs.: Volklichkeit. - Präd. und Synt. (zum Subjekt): die Demokratie [...] verletzte den Genius der Nation; (mit und in einer präp. Angabe oder Ergänzung:) wir dürfen Volklichkeit ${ }^{* 14}$ nicht mit Demokratie verwechseln // an der Demokratie zu Grunde gehen // durch Demokratie untergehen; (in präp. Attr.:) Schein von Demokratie war in Wirklichkeit nur Dilettantismus / ein Anflug von Liberalismus; (mit Attr.): absolute / formale Demokratie.

Belege: [...]

\section{demokratisch, Adj}

1. allgemein und wertneutral: >sich auf eine Herrschaftsform bzw. ein politisches System beziehend, das den Willen der Mehrheit des Volkes respektiert bzw. in dem das Volk an der Machtausübung im Staate teilhat und das den Willen des Volkes repräsentiert; auf einem solchen System beruhend, nach ihm verfahrend, ein solches anstrebend<; offen zu 3; vgl. Demokratie 1.

2. völkisch und national konnotiert: >sich auf eine Herrschaftsform bzw. ein politisches System beziehend, das den Willen des Volkes zum Handlungsmaßstab erhebt, diesen als Kollektivwillen ansieht und dabei mit besonderer Komponente des Verknüpft- und Eingebundenseins in ein ständisch organisiertes führerbezogenes System den Willen des Einzelnen bzw. einzelner Gruppen ausgrenzt, da dieser nicht dem Wohle der Gemeinschaft dienen kann<; vgl. Demokratie 2. - Bdv.: konservativ. - Paraph.: für das Volk zu handeln // das Aufgehen und die Hingabe zum Volksstaat.

3. liberal pluralistisch konnotiert: >sich auf eine parlamentarische und republikanische Herrschaftsform mit einem pluralistischen Parteiensystem beziehend, das den Willen der Mehrheit repräsentiert und durchführt bzw. in dem das Volk an der Machtausübung vermittels freier Wahlen und dadurch gewählter Repräsentanten teilhat; auf einem solchen System beruhend, nach ihm verfahrend, ein solches anstrebend<; vgl. Demokratie 3. - Bdv.: republikanisch / liberal / freisinnig / fortschrittlich / parlamentarisch / pazifistisch / sozialdemokratisch / undemokratisch / westlerisch. -Paraph.: nicht zu handeln. - Ggb.: monarchisch, konservativ.

Schon ein kurzer Blick auf die Artikel Demokratie bzw. demokratisch zeigt, dass beide Ausdrücke polysem sind, und das in analoger Weise. ${ }^{15}$ Wir haben eine wertneutrale bis positive allgemeine Bedeutung, Bed. 1, eine weitere ausgesprochen positiv bewertete Bedeutung, Bed. 2, und eine dritte Bedeutung, die im Gegensatz zu 1 und 2 vollkommen negativ konnotiert ist. Generisch ist Demo-

14 Das Sternchen zeigt an, dass im Originaltext ein Pronomen stand, das nun durch das Bezugswort ersetzt wird.

15 Die innere Parallelităt der Bedeutung von Demokratie und demokratisch wird mittels semantischer Kommentare des Typs vgl. Demokratie $l$ hergestellt. 
kratie auch für Moeller ein Terminus, der Prestigewert besitzt. Und in einem generischen Sinne bedauert auch er, dass die „Demokratie von den anderen Völkern verraten wurde" und dass ,sich das Westlertum für Demokratie ausgibt“. Die letzte Aussage suggeriert deutlich, dass Moeller Demokratie in einem generischen Sinne nicht als etwas Negatives interpretiert. Anders ausgedrückt: Moeller könnte sich durchaus mit einer von ihm als demokratisch bezeichneten Staatsform anfreunden. Was er jedoch ablehnt, ist die Art und Weise, wie Demokratie, speziell die Weimarer Demokratie, umgesetzt wird. Die (in doppeltem Sinne) rechte Demokratie spiegelt sich in Bed. 2. Zu ihr gehört eine konservative und nationalistische Grundhaltung, die ständestaatlich organisiert ist und den Willen des Volkes nicht als Sammelsurium vieler ungerichteter Einzelwillen versteht, sondern als zugleich einheitlichen wie gegliederten kollektiven Willen. Kontextcharakteristische Wörter, also Wörter, die den kommunikativen Rahmen prägen, sind dabei die Gemeinschaft, die Genossenschaft, vor allem jedoch die unter den Bedeutungsverwandtschaften zu findenden Ausdrücke Volklichkeit und Bindung. Im Gegensatz hierzu ist in Bedeutung 3 das Individuum maßgeblich. Diese pluralistische, international orientierte und vor allem liberaldemokratische Ausprägung kann Moeller nicht akzeptieren und verurteilt sie als Demagogie, als Schiebertum und windigen Halbintellektualismus. Bei den Paraphrasen findet man auf einen Blick: liberales Chamäleon, Moloch, der Massen und Klassen und Stände und Unterschiede des Menschlichen frisst, Leviathan usw. Bedeutungsunterscheidend zwischen 2 und 3 ist die Tatsache, dass letztere Bedeutung liberale, erstere konservative Züge hat, was sich in den Bedeutungsverwandtschaften besonders beim Adjektiv demokratisch demonstrieren lässt; vgl. konservativ zu demokratisch 2 und sozialdemokratisch, westlerisch, liberal, freisinnig usw. zu demokratisch 3. Moeller geht es um Polarisierungen und die Schaffung einer geordneten, überschaubaren Weltstruktur, in der politische Eindeutigkeit gefordert wird. Die Konzepte Konservativismus / konservativ und die Konzepte liberal und Liberalismus bilden die beiden sich diametral gegenüberstehenden Pole dieser Welt. Es wird dabei deutlich, dass die Frage, ob Demokratie oder nicht, für Moeller nahezu marginal ist. ${ }^{16}$ Moeller macht in seinem Buch nicht die Demokratie zum Gegner, sondern den Liberalismus.

Der Liberalismus ist also das eigentliche politische Feindbild Moellers. ${ }^{17}$ Bei der Analyse wurde das Wort monosem interpretiert, obwohl man durchaus diskutieren könnte, ob nicht besser drei Bedeutungen angesetzt werden sollten.

16 Moeller van den Bruck 1923, 284: „Er [der konservative Mensch] weiß nur nicht - ob wir nicht durch Demokratie untergehen werden. Er selbst ist ohne die Nebenabsichten und Hintergedanken der Parteipolitik. Seine Partei ist Deutschland. Die Staatsformen dagegen: Republik, Diktatur, Nationalverteidigung, [...] welche es nun sein mögen, welche uns noch bevorstehen sie sind für diesen konservativen Menschen nur Mittel zum Zweck Er ist heute konservativ nicht um des Staates willen, sondern um der Nation willen. Und die Macht des Staates, ohne die für ihn Staat nicht zu denken ist, wird von ihm nur noch um der Freiheit des Landes willen gewollt."

17 Vgl. dazu: Marcuse 1965,21 
Die Belege erwiesen sich jedoch bei der praktischen Analyse als kaum unterscheidbar, was darauf hindeutet, dass der Autor alle drei Inhaltskomponenten immer gleichzeitig meint. Der Unterscheidung der Nuancen halber habe ich aber die inhaltlichen Varianten in a, b und $\mathrm{c}$ untergliedert. Liberalismus ist also im Sprachgebrauch Moellers:

\section{Liberalismus, der.}

a) >verkommene, hinterhältige und betrügerische Geisteshaltung, die sich in Schmarotzertum, Opportunismus, Bindungslosigkeit, Individualismus, Egoismus äußert, Charakterlosigkeit, die das eigene Interesse und die eigene Profitgier über das Wohl der Nation stellt $<$; b) >mangelnde geistige Kompetenz, Torheit, die durch die westliche Aufklärungsphilosophie geprägt ist und das innerhalb der deutschen Nation Realisierbare nicht mehr erkennen kann, sich der Selbsttäuschung hingibt und dadurch auch alle anderen klaren Positionierungen verwischt $<$; c) >zersetzerische, alle Bindungen zerstörende Weltanschauung, die die Position bzw. Partei der Geschäftemacher, der verschwörerischen Freimaurer und des Feindes vertritt, den Krieg verursacht und das Vaterland verraten hat<. - Bdv.: Betrug, Taktik, Trivialität, Intellektualismus, Intrige, Vermittlertum, Westlertum, Nutznießertum, Demokratie, Demagogie, Individuum, Auflösung, Fortschritt, Erkrankung, Zersetzung, Parlamentarismus, Rationalismus, Utilitarismus, Vernunft, Schwindel, Willkür. - Paraph.: Freiheit, keine Gesinnung zu haben // ein Netz, Geflecht und Schlingenwerk, das man sich durch die Welt hin ausgelegt vorstellt und in dessen Maschen sich Deutschland schließlich verfangen soll // Ausdruck einer Gesellschaft, die nicht mehr Gemeinschaft ist / die sich aus minderen Bestandteilen des Volkes zusammensetzt // Partei der Emporkömmlinge // die Partei einer Zwischenschicht, die verstanden hat, sich zwischen das Volk und die Auslese einzuschieben "| Selbstauflösung des Menschen "| politischer Trick /" Element der Auflösung // beschützte Willkür // Geistige Ansteckung, deren Träger sich durch Unempfindlichkeit zu schützen pflegen, während sie die Befallenen völlig zu Grunde richtet // Schwindel, der mit Ideen getrieben wurde // moralische Erkrankung. - Ggb.: Bindung, Gemeinschaft, Konservativismus, deutscher Sozialismus, Nationalismus; Ggsparaph.: Welt als einheitliches Erlebnis, das der Mensch mit dem Menschen teilt. Präd. und Synt. (zum Subjekt): der Liberalismus hat [...] zersetzende Wirkung / schleppt den liberalen Toren mit sich / beruht auf dem liberalen Menschen / ist nicht an eine bestimmte Partei gebunden / führt zu Dummheit oder zu Verbrechen / ist die Partei der Emporkömmlinge / hat die Kulturen untergraben / hat Religionen vernichtet / hat Vaterländer zerstört / hat alles, auch die Monarchie zerstört / ist die Selbstauflösung des Menschen / lebt vom Zeitlichen / entzieht sich den Problemen der Nation; (als Akk. Obj.): das Ich hat sich den Liberalismus geschaffen; (mit präp. Angabe oder Ergänzung): an Liberalismus gehen die Völker zu Grunde // der Konservative sieht im Liberalismus den Ausdruck einer individualistischen / egoistischen Lebensanschauung; (als Attr.): Geistesverfassung der Freimaurer kehrt als Geistesverfassung des Liberalismus wieder // die zersetzende Anschauungswelt eines Liberalismus bringt Auflösung / verbreitet moralische Erkrankung der politischen Völker / verdirbt den Karakter derselben Völker // das größte Verbrechen des Liberalismus war seine große Dummheit // der Westen ist die Heimat des Liberalismus; (Teil eines Präpositionalattributes:) der Kampf gegen die Aufklärung wird ein Kampf gegen den Liberalismus; (mit Attr.): deklamierter, dummer, starrer, rechthaberischer, rationalistischer, über- 
heblichster, ungebundener, unbedenklicher volkloser, wahlloser L., der nationalistisch gewordene L.; ein geflissentlich arbeitender L.; Liberalismus eines Westlertums.

Oder im Artikel liberal:

liberal, Adj.

a) >charakterlich verkommen, opportunistisch, egoistisch, profitgierig, landesverräterisch, hinterhältig, verantwortungslos, prinzipienlos, national bindungslos<; b) $>$ intellektuell kurzsichtig, dumm, unbelehrbar<; c) >weltanschaulich, zersetzerisch, nationale Werte missbrauchend und verderbend, das Individuum über die Gemeinschaft stellend und damit die Gemeinschaft und die Nation verratend <. - Bdv.: westlich, parlamentarisch, verfassungspolitisch, verräterisch, abwendig, revolutionär, sozialistisch, aufklärerisch, westlerisch gerichtet, opportunistisch, weltkapitalistisch, freisinnig, fortschrittlich, zivilisiert; - Paraph.: jd., der das Ziel, auf das er hinarbeitet, hinter dem Vorbehalte versteckt, es von vornherein je nach seiner Erreichbarkeit zu formulieren // Mensch mit einem je nachdem aufgeweichten oder abgefeimten Gehirne, der entweder seine Grundsätze nicht mehr auseinanderzuhalten vermag oder der sich über sie hinwegzusetzen pflegt: ein* Mensch jedenfalls, den es nicht die geringste Selbstüberwindung kostet, sie preiszugeben, im Gegenteile, der sich daraus bezahlt macht und sich dabei in seinem eigentlichen Geschäfte fühlt / der immer und überall in der ihm eigentümlichen Weise handeln wird, und der, wo und wie er auch wirken möge, immer zersetzend wirkt // jeder Mensch, der sich nicht mehr in der Gemeinschaft fühlt. Ggb.: konservativ, magisch. - Präd. und Synt. (attributives Vorkommen): der liberale Mensch: ${ }^{18}$ ist mit den Werten nach seinen Launen herumgesprungen / sucht mit Kälte / mit Zugriff / mit Bedenkenlosigkeit seine Absichten zu erreichen // ist irgendwie jeder Mensch, der sich nicht mehr in der Gemeinschaft fühlt / ist ein durchaus gewöhnlicher Mensch / ist politischer Individualist / ist Opportunist aus System / ist unbelehrbar wie immer / ist der Feind des konservativen Menschen / ist immer Bejaher // der liberale Mensch hat Werte verfälscht / hat das Schlagworte der Demokratie herausgegeben / hat Grund, irgendwelche Dinge, die sich aber nicht verbergen lassen, zu verbergen / hat die Forderung, dass das deutsche Volk politisiert werden müsse, gestellt // schiebt Verantwortung ab // trägt überall Zersetzung hinein // konnte ein ganzes Volk auf der Ebene der Außenpolitik ausbeuten // drückt keine gegliederte Gesellschaft aus // drückt eine aufgelöste Gesellschaft aus // kann keine Werte hervorbringen, die dem Volke und der Gesellschaft gemeinsam wären // riß diese entsetzliche Trennung auf, die zwischen Angehörigen desselben Volkes klafft // frißt jede Schande, die man ihm hinwirft // verdankt seine Stellung nicht der eigenen Kraft // spielte die Linke gegen die Rechte aus // sieht nicht, daß ein Volk erst dann politisiert werden kann, wenn es nationalisiert ist // lernt gar nichts; (als Attr. im Dativobjekt:) dem liberalen Menschen unterlaufen immer wieder Dummheiten // ist das Volk gleichgültig; (als Attr. mit einer präp. Angabe oder Ergänzung): Freiheit ist für den liberalen Menschen der Spielraum, den er sich für seinen Egoismus zu schaffen weiß; (als Attr. im Genitivattr.): Allzumenschlichkeiten / Aufstieg / Eigentümlichkeit / Halbund Zwischenbegriffe / Selbstsucht / Trieb / Unbelehrbarkeit des liberalen Menschen;

18 Aufgrund der Tatsache, dass der liberale Mensch so häufig erscheint, wurden die vorkommenden Belege an den Anfang gestellt und intern noch einmal nach dem grammatischen Vorkommen innerhalb der Kollokation gegliedert. 
(liberal + Person bzw. Kollektiv): der liberale Tor // der Kaiser war ein liberaler Monarch; (liberal + Gegenstand bzw. Sachverhalt): die deutsche Revolution bleibt eine liberale Revolution // es gibt eine liberale Vertrottelung [die jeden Gedanken gutheißt, wenn er nur ",frei", und doppelt gut, wenn er außerdem noch ", nützlich "ist] /I liberale Unzuverlässigkeit // die schrankenlose Herrschaft eines liberalen Staatsideals ist zum revolutionären Staatsideal geworden // der deutsche Sozialismus ist den liberalen Unentschlossenheiten verfallen / Demokratie ist ein liberales Chamäleon // das liberale Schmarotzertum // die Revolution war ein liberales Zwischenspiel; (prädikatives Vorkommen): die politische Partei ist liberal // der Reichstag, der [...] ein liberal durchseuchter Körper ist // die Einfältigen / die Zweideutigen sind liberal // das Genie des deutschen Volkes ist konservativ / nicht liberal / nicht revolutionär; [...].

\section{Belege: $[\ldots]$}

Die drei Ebenen werden deutlich. Liberalismus/liberal sind für Moeller Eigenschaft, Kompetenz und daraus resultierende Weltanschauung. Ähnliches gilt für Konservativismus und konservativ, dort allerdings mit genau entgegengesetztem Vorzeichen. Die zu Konservativismus angesetzte Bedeutung lautet:

\section{Konservativismus, der}

a) >metapolitische und überzeitliche Geisteshaltung, die in einem patriotisch pflichtbewussten, verantwortungsbewussten Charakter besteht und sich zum Ziele gesetzt hat, das für die eigene Nation Erhaltenswerte, die nationalen Werte zu bewahren, im Ernstfall zu verteidigen bzw. zu retten<; b) >intellektuelle Fähigkeit, höhere Einsicht in die Geschichtlichkeit als solche und in die der deutschen Nation im Besonderen, in die Zukunft, den Charakter, das Wohlergehen des deutschen Volkes; Kenntnis darüber, was für das Wohlergehen der Nation erhaltenswert ist<; c) >nationalpolitische Weltanschauung, Utopie, die auf einen starken deutschen Staat mit einer starken Führerpersönlichkeit an der Spitze und einem starken Kollektivbewusstsein abzielt, so dass der einzelne Mensch sich der Politisierung entziehen kann bzw. dass im Ganzen des Staatsgefüges die Gemeinschaft über dem Individuum steht<; metonymisch: > politische Bewegung $<$; in organisierter Form: $>$ Partei $<$. Bdv.: Karakter. - Paraph.: organische natürliche volkliche Grundlage // konservative Verstand, der die Begebenheiten aus dem Ganzen versteht // Herrschaft des Staates, die Verpflichtung der Berufsstände, die Bildung einer Führerschicht. - Ggb.: Liberalismus. - Präd. und Synt. (zum Subjekt): der Konservativismus verträgt keine Kompromisse // hat die Ewigkeit für sich // beruht auf Menschenkenntnis // ist Wurzelanschauung // verlässt sich auf das Ewige // ist der Verstand einer Nation // hat das Problem der Nationalität zu einfach genommen // setzte das urkonservative Werk nicht fort // ist ein Lebendiges - kein Totes - im Ewigen // lässt sich einem Volke mitteilen // Konservativismus und Staat sind gleichbedeutend; (als Attr.): Gesetz des schöpferischen Konservativismus // Weltanschauung des Konservativismus gründet sich auf Verantwortung; (mit Attr.): bürokratischer / schöpferischer / theologischer Konservativismus.

Belege: $[\ldots]$ 
Bedeutung von konservativ:

konservativ, Adj.

a) bezogen auf eine moralische, charakterliche Geisteshaltung: >patriotisch, nationalistisch, pflicht- und verantwortungsbewusst, stetig, bewahrend, dem Ewigen und Geschichtlichen verbunden<; b) in Bezug auf eine kognitive Kompetenz: >wissend, verstehend, weitsichtig, klarsichtig, verständig, im Überzeitlichen, Ewigen denkend, in Zusammenhängen, besonders in nationalen denkend, das Große und Bedeutende im Auge habend, realistisch<; c) >einer weltanschaulichen Richtung angehörend, die metapolitisch ist und das durch eine gesellschaftliche Politisierung verlorengegangene nationale Wertesystem eines ganzheitlichen Staatsdenkens, wenn notwendig, revolutionär wieder erringen möchte<. - Bdv.: geschichtlich, revolutionär, überzeitlich, ewig, erhaltend, nichtproletarisch, friderizianisch, nationalistisch, stetig. - Paraph.: in Gesetzmäßigkeiten zu denken, die sich immer wieder herstellen // immer wieder einen Anfang zu setzen // Dinge zu schaffen, die zu erhalten sich lohnt. - Ggb.: fortschrittlich, zeitlich, liberal, primitiv, materialistisch, reaktionär, unkonservativ, individualistisch, egoistisch. - Ggsparaph.: sich mit Erwartungen zu beschäftigen, die sich niemals erfüllen. - Präd. und Synt.: (Attribut im Subjekt) der konservative Mensch (Subj.) ist der Mensch, der auf alle Möglichkeiten gefasst war / ist nicht dazu da, um zu versagen, wenn alles versagt, sondern dazu da ist, um sich zu bewähren, wenn sich niemand bewährt / ist der gegründeten Ansicht, daß unsere ganze Zeit irre gegangen ist / ist notwendig Erhalter und Empörer zugleich / ist berufen, den Massen die Persönlichkeit entgegenzusetzen / ist erfahren im Zeitlichen / im Ewigen / ist gewöhnt, Ansprüche an den Menschen zu stellen / ist ohne die Nebenabsichten und Hintergedanken der Parteipolitik / ist konservativ nicht um des Staates willen, sondern um der Nation willen // weiß, daß die Welt immer wieder wird, was sie von Natur ist / daß die Welt, die bis zu dem Augenblicke konservativ gewesen ist, in dem der liberale Mensch in sie eintrat, erst dann wieder konservativ werden kann, wenn der liberale Mensch ausgetilgt worden ist // erkennt hinter der Revolution einen geistigen Vorgang / die vermittelnde Macht, die Vergangenes an Künftiges weitergibt / mitten im Seienden das Bleibende / das Überdauernde / die Gefahr // sieht seine Zweifel, die er von Anbeginn dem Systeme des Parlamentarismus entgegenbrachte, bestätigt / sieht den geschichtlichen Vorgang / sieht die Gegenwart nach der Seite der Zukunft geöffnet / sieht die Welt, wie sie immer sein wird // denkt überzeitlich in den ewigen Gegebenheiten der menschlichen Natur / denkt an das dritte Reich // versteht die Wirkungen aus ihren Ursachen / verstand (ihn / Sozialismus) von der Gruppe von der Gemeinschaft, von der körperschaftlichen Bindung der Nation aus // durchschaut diesen Schwindel sucht jetzt wieder anzuknüpfen nicht abzubrechen - wie der revolutionäre Mensch // gibt sich eine Rechenschaft über Zusammenhänge / über alles, was flüchtig ist / gibt sich keiner Selbsttäuschung darüber hin, daß [...] // steht als Wächter dieser Werte // hat ein Zeitempfinden, das ihm diese Probleme unmittelbar vertraut macht // hat ein Weltbild, in das sie [die Probleme der Revolution] sich in ihrer Bedeutung oder Nichtbedeutung abermals einfügen // hat eine verwurzelte Auffassung von Geschichte // hat noch das Blut der Männer, die einst aufgebaut haben in sich // besitzt diesen Karakter [sich keinen Selbsttäuschungen hinzugeben] nebst der körperlichen Tüchtigkeit und der sittlichen Entschlußkraft, aus dem Karakter $z u$ handeln, sobald es von Ihm verlangt wird // besitzt von seiner Überlieferung her das konservative Wissen, daß alle Scheinfriedlichkeit der Welt nicht über die Wirklichkeit täuschen kann // lebt im Bewußtsein der Ewigkeit, die alle 
Zeitlichkeit umschließt / daß Geschichte ein Erbe ist, eine große Weitergabe / lebt in einer revolutionären Teilhaftigkeit, die ihm im Gegensatze zum reaktionären Menschen erst das Recht gibt, nicht für die Revolution zu sein, sondern wider sie // stellt seine große und räumliche Sehweise über den kleinen und zeitlichen Gesichtspunkt // bringt von Hause aus die Gabe mit, zu urteilen und zu schließen, zu erkennen was wirklich // überwand den mechanischen Sozialismus, [...] durch einen organischen Sozialismus der Praxis werden sollte // löste den atomisierenden Sozialismus durch einen korporativen Sozialismus ab // wendet sich nicht an den Parteideutschen, in dem er, ob er ihm nun auf der Linken, ob er ihm nun auf der Rechten begegnet, das Unheil der Nation in seinen Gründen verkörpert sieht; (Attr. im Genitivattribut): Führung ist [...] ein ewiges Vorrecht des nichtproletarischen Menschen, nicht des demokratischen Menschen, doch des konservativen // der reaktionäre Mensch ist eine Entartungsform des konservativen Menschen // der liberale Mensch ist des konservativen Menschen Feind // die Stunde, in der sich diese Freiheit entscheidet wird die Stunde des konservativen Menschen sein; (Attr. im Dativobjekt:) dem konservativen Menschen ist die Nation der Inbegriff alles dessen, was er vom Menschen weiß, was er auf der Erde besitzt, wohinein er seinen Willen legen und wofür er im Leben tätig sein kann; (Attribut in einer präp. Angabe oder Ergänzung:) [...] // Für den konservativen Menschen ist jene Vereinbarung des konservativen Staatsgedankens mit dem demokratischen kein Problem // ist die Staatsformen dagegen: Republik, Diktatur, Nationalverteidigung, oder welche es nun sein mögen, welche uns noch bevorstehen nur Mittel zum Zweck; (konservativ + Person bzw. Kollektiv): der konservative Deutsche einer dritten Partei stößt zwischen allen parteipolitischen Gegensätzen durch / wendet sich jenseits von einem politischen Denken das Deutschland und das Europa verdarb, überall an den Menschen im Deutschen, und an den Deutschen im Menschen; (konservativ + Gegenstand bzw. Sachverhalt): konservative Politik ist große Politik // der konservative Gedanke, nicht der liberale konnte uns retten // das konservative Denken sieht in allen menschlichen Verhältnissen ein Ewiges wiederkehren // der konservative Verstand ist der einzige, der die Begebenheiten aus dem Ganzen versteht // das konservative Wissen, daß [...] // die Revolution bestätigte das große konservative Lebensgesetz // die konservativrevolutionäre Gegenbewegung des Nationalismus* will das Leben der Nation; (prädikatives Vorkommen): was heute revolutionär ist, wird morgen konservativ sein // das Genie des Deutschen Volkes ist konservativ // die Welt ist von Natur aus konservativ // der Nationalismus ist konservativ.

Betrachtet man nun die Syntagmenangaben der Adjektive, so ergibt sich zunächst einmal, dass Moeller den konservativen Menschen dem liberalen gegenüberstellt. Tatsächlich ergibt der Belegbefund eine außerordentliche Häufigkeit an Stellen, in denen er von ,dem liberalen“ oder von „dem konservativen Menschen“ schreibt, das heißt eine politische und gleichsam anthropologische Typisierung vom Menschen vornimmt. Bei liberal lässt sich dies nahezu bei der Hälfte der Belege aufzeigen. Konservativ und liberal beziehen sich somit deutlich auf entgegengesetzte menschliche Eigenschaften, Kompetenzen und ihre Folgen. Moeller geht es nicht primär um politische Einordnungen, sondern darum, das Politische ins Metapolitische, Geistige bzw. Charakterliche zu überhöhen. Um so negativer und umfassender ist dann auch seine Beschreibung des liberalen Menschen und der dazugehörigen Abstraktionsgröße ,Liberalismus'. Ich greife 
der Pointierung halber einige der Syntagmen aus den Artikeln heraus, in denen deutlich wird, welche charakterlichen oder intellektuellen Schwächen dem liberalen Menschen von Moeller van den Bruck zugewiesen werden.

„Der liberale Mensch sucht mit Kälte / mit Zugriff / mit Bedenkenlosigkeit seine Absichten zu erreichen;

Der liberale Mensch hat sich auf der Ebene der Innenpolitik bis dahin schadlos gehalten;

Der liberale Mensch konnte ein ganzes Volk auf der Ebene der Außenpolitik ausbeuten;

Der liberale Mensch ist irgendwie jeder Mensch, der sich nicht mehr in der Gemeinschaft fühlt;

Der liberale Mensch ist Opportunist aus System;

Der liberale Mensch ist unbelehrbar wie immer;

Der liberale Mensch frißt jede Schande, die man ihm hinwirft;

Dem liberalen Menschen unterlaufen immer wieder Dummheiten;

Dem liberalen Menschen ist das Volk gleichgültig.“

Im Gegensatz dazu kann man über den konservativen Menschen lesen:

„Der konservative Mensch ist berufen, [diese Werte] vor Einebnung durch Demokratie wie Proletariat zu schützen;

Der konservative Mensch versteht die Wirkungen aus ihren Ursachen;

Der konservative Mensch denkt überzeitlich in den ewigen Gegebenheiten der menschlichen Natur;

Der konservative Mensch findet sein Ich in der Nation als Gemeinschaft wieder;

Der konservative Mensch ist der Mensch, der auf alle Möglichkeiten gefasst war;

Der konservative Mensch lebt im Bewußtsein der Ewigkeit, die alle Zeitlichkeit umschließt;

Der konservative Mensch sieht die Welt, wie sie immer sein wird;

Der konservative Mensch ist erfahren im Zeitlichen // ist erfahren im Ewigen;

Der konservative Mensch stellt seine große und räumliche Sehweise über den kleinen und zeitlichen Gesichtspunkt;

Der konservative Mensch ist ohne die Nebenabsichten und Hintergedanken der Parteipolitik;

Der konservative Mensch ist konservativ nicht um des Staates willen, sondern um der Nation willen;

Der konservative Mensch besitzt diesen Karakter [sich keinen Selbsttäuschungen hinzugeben] nebst der körperlichen Tüchtigkeit und der sittlichen Entschlußkraft, aus dem Karakter zu handeln, sobald es von Ihm verlangt wird.“

Dass Moeller van den Bruck hier eine ausgesprochene Schwarzweißmalerei betreibt, ist offensichtlich. Seine Aussagen sind dogmatisch, nicht argumentierend oder abwägend. Mit der Typisierung einhergehend ist die Verallgemeinerung desjenigen, was am wenigsten überprüfbar ist. Moeller enthebt sich so der Überprüfung oder der Diskussion und bietet dem Leser eine klar geordnete ewige Weltschau, die in einer Welt, die im Chaos zu versinken droht, wie es nach dem 
Weltkrieg der Fall war, nahezu heilsversprechend ist. Er hat den Feind ausgemacht und auch die Opfer, die in eigenartiger Verknüpfung auch gleichzeitig die Helden der Zukunft darstellen. Dass sie noch nicht stark genug sind, nicht schon längst ihre Kompetenz bewährt haben, ist wiederum die Schuld des Feindes, der eben verschwörend im Hindergrund wirkt. Diese Beobachtung wird mit einem Blick auf die verschiedenen Verbarten noch unterstrichen. Weil dem konservativen Menschen ein tiefes, ein witterndes, ein unbeirrbar richtiges Gefühl für den Zusammenhang der Dinge / das aus einer erprobten Überlieferung kommt und obendrein den Vorzug brutal-natürlicher Verständigkeit hat, angeboren ist, sieht, denkt, erkennt, versteht, und weiß er, was die Notlage ist, wer der Schuldige ist und was deswegen getan werden muss. Weil er diese Kompetenzen hat, ist er sogar berufen, das Schicksal der Nation in seine Hand zu nehmen.

Die hier verwendeten Verba noscendi bzw. percipiendi fallen um so mehr ins Gewicht, wenn man sie mit denjenigen Verben vergleicht, die in Verbindung mit liberaler Mensch gebraucht werden. Dort gibt es sie höchstens dann, wenn der Autor darauf hinweisen will, dass der liberale Mensch sich nur in Ausbeutung und Betrug auskennt: denn Freiheit ist für den liberalen Menschen nur der Spielraum, den er sich für seinen Egoismus zu schaffen weiß. Der liberale Mensch hofft lediglich, glaubt und behauptet oder leugnet. Ihm unterlaufen Dummheiten und er verdreht alles. Doch er verfehlt seine Ziele, insgesamt alles, was dem Wohle der Nation dient. Dass dies nicht nur aufgrund mangelnder Kompetenz geschieht, sondern letztlich auf charakterliche Mängel zurückzufuihren ist, stellt man schnell fest, wenn man alle weiteren Verben heranzieht, denn Moeller van den Bruck gebraucht in Bezug auf den Liberalen besonders häufig Verben wie: ausbeuten, sich schadlos halten, die Verantwortung abschieben, verfälschen, aufreißen, missbrauchen, verbergen, verdecken, j. gegen $j n$. ausspielen. Wie gefährlich ein solcher Charakter für die Volksgemeinschaft sei, zeigt wiederum ein kurzer Blick auf die Paraphrasen, denn Liberalismus ist: eine geistige Ansteckung, deren Träger sich durch Unempfindlichkeit zu schützen pflegen, während sie die Befallenen völlig zu Grunde richtet / eine moralische Erkrankung. ${ }^{19}$ Moellers Metaphorik wirkt suggestiv. Liberalismus als ansteckende Krankheit zu bezeichnen, nimmt die Distanz aus der Beobachtung, schafft Bedrohlichkeit und ist handlungsauffordernd. Die Einteilung der Welt in richtig und falsch, die Polarisierung in Gute und Böse ist das eine Ergebnis seiner Ausführungen, doch mit der Diskriminierung des Liberalismus als politisch inkompetent gibt er sich nicht zufrieden. Weltanschauung und Person, die diese Weltanschauung hat, sind bei Moeller nicht zu trennen. Es genügt ihm nicht, gegen den Liberalismus zu schreiben, da er damit nur den Bazillus bekämpfen kann. Den bereits infizierten Träger, den liberalen Menschen also, der den Krankheitskeim in sich trägt und vor allem weiterträgt, den erreicht er nicht. Er will aber die absolute Isolierung des Feindes, da sonst Ansteckung und Untergang für alle droht.

19 Vgl. dazu auch: „Liberale Menschen sind niemals magische Menschen. - Liberalismus führt zur Dummheit oder zum Verbrechen“. Moeller van den Bruck 1923, 111. 
Anhand der Syntagmen würden sich auf einen Blick noch viele weitere Linien nachzeichnen lassen, die hier nur angedeutet werden können, so der immer wieder aufgenommene Topos vom liberalen ,Schmarotzertum ' und nicht zuletzt die liberale Verschwörungstheorie, ${ }^{20}$ die von Moeller als Tatsache hingestellt und sowohl außenpolitisch als auch innenpolitisch als äußerst erfolgreich gekennzeichnet wird. Einem Bazillus gleich habe der Liberalismus alles zersetzt oder wie von einem Maulwurf, der in einem gut gepflegten Garten wütet, wurde von ihm alles unterwühlt, so sehr, dass sogar der gesunde Konservativismus ins Wanken geriet. Man fühlt sich deutlich an den Sprachgebrauch des Nationalsozialismus erinnert.

Liberal bezeichnet demnach, wie oben schon gesagt, sowohl charakterliche Defizienz, die der Nation zum Schaden gereicht, als auch Dummheit. Beide Zuschreibungen zusammen ergeben eine Weltanschauung, die für Moeller van den Bruck als Gegenpol zu ,konservativ' letztlich nur Verderben für die deutsche Nation und deren Fähigkeiten bringen kann. Insgesamt wird der Liberalismus für alles Schlechte verantwortlich gemacht, das den Deutschen seit Bismarck widerfahren ist.

Dementsprechend wäre die Charakterisierung, antiliberales Denken' für Moeller van den Bruck und damit für den Neukonservativismus zutreffender als antidemokratisch. Denn antiliberal umreißt nicht nur die Abneigung gegen Wirtschaftsliberalismus, Aufklärung und Individualismus, sondern geradezu wörtlich: die Abneigung gegen alles freisinnige und kritische Denken, gegen Einzelindividuen und außerhalb der Gemeinschaft stehende Gruppen und damit radikalste nationalchauvinistische Intoleranz, die den Boden für den Nationalsozialismus geebnet hat.

Und hier findet man dann auch das eigentliche Bindeglied zwischen den Nationalsozialisten und Moeller. Der Begriff, der sich nach genauerer lexikologischer und semantischer Betrachtung in allen Artikeln erarbeiten lässt, ist deutlich der des ,Nationalen'. Denn stellt man die Frage nach dem verbindenden bzw. trennenden Kriterium zwischen den Einzelbedeutungen der Einzelwörter, so wird deutlich, dass das Kriterium für positive Bewertung ebenso wie für die negative Bewertung dasjenige der nationalen Orientierung ist. Nationalismus ist das eigentliche Programm, die alles durchdringende Ideologie Moellers. ${ }^{21}$

20 Moeller van den Bruck 1923, 114.

21 Moeller van den Bruck 1923, 329f:: „Über Deutschland weht heute nur eine Fahne, die Zeichen von Leid und ein Gleichnis unseres Daseins ist: nur eine einzige Fahne, die keine Farben neben sich verträgt und den Menschen, die in ihrer Düsterkeit gehen, alle Lust an bunten Wimpeln und frohen Standarten nimmt: nur die schwarze Fahne der Not, der Demütigung und einer letzten Erbitterung, die Gefaßtheit ist, um nicht Verzweiflung zu sein - Banner der Unrast von Gedanken, die bei Tage und in der Nacht um das Schicksal kreisen, das eine verschworene Welt unserem entwaffneten Lande zugedacht hat - Banner des Widerstandes von Männern, die nicht in Ergebung ein Vernichtungswerk hinnehmen wollen, das mit der Zerreißung des Landes beginnen und mit der Austilgung unseres Volkstums enden soll - Banner, des Aufbruches von Deutschen, die entschlossen sind, den Betrug auf die Betrüger zuruckzulenken, die Nation zu retten und das Reich zu bewahren. [...] Man nennt diesen Willen heute nicht konservativ. Man nennt ihn nationalistisch. Aber er will die Erhaltung von allem, was in Deutschland erhaltenswert ist. Er will 
Zur Demonstration sei hier der Artikel national vorgestellt.

national, Adj.

$>$ das Wohl des eigenen Volkes betreffend, es in besonderer, radikaler Weise in das Zentrum des Interesses stellend, es fördernd; einem Volke eigentümlich, zugehörig $<$; speziell: >innenpolitisch orientiert<. - Bdv.: deutsch, erdverwurzelt, heimatverbunden, jungsozialistisch, konservativ, mystisch, patriotisch, politisch, radikal, staatlich, volkverwurzelt. - Paraph.: Vision von den Dingen // Volklichkeit, Bindung, lebensgeschichtliche Verbundenheiten // vor allem an das eigene Volk denken // zu der Sache Ihres Vaterlandes rein und unbedingt und überschwenglich wie Nationalisten stehen // im Bewußtsein seiner Nationalität leben / im Bewusstsein seines Staates leben. - Ggb.: franzosenfreundlich, heimatlos, international, liberal, ungebunden, volklos, wahllos. Ggsparaph.: Internationale, in der die Unterschiede der Völker und Sprachen, Rassen und Kulturen aufgehoben, ausgemerzt und gänzlich verwischt sind I" auf Völkerverbrüderung und ewigen Frieden gerichtet. - Präd. und Synt.: (national + Gegenstände und Sachverhalte:) Der Parlamentarismus schwächt die nationalen Leidenschaften nach Möglichkeit ab // Wir haben die Möglichkeiten eines unfertigen, aber auch unerschöpften Volkstums voraus, das noch nicht zu seinem nationalen, geschweige denn politischen Ich kam // ob wir fähig werden, unserem politischen Dasein die nationale Gestalt zu geben // ob wir nicht der völligen nationalen Vernichtung entgegengehen // Die Revolutionsdemokratie* verfolgte die nationale Opposition // daß die Revolution eines Volkes nur eine nationale Revolution sein kann // Marx schüttete die Keime eines nationalen Sozialismus zu // der Rationalist, war ganz ohne das mystische Erlebnis, und war ohne das nationale Erlebnis // wir brachen unsere Geschichte durch eine Revolution vorläufig ab, die nicht so sehr eine nationale Revolution, als eine internationale [...] Revolution sein sollte // Wille zur Demokratie ist Wille zum politischen Selbstbewußtsein eines Volkes. und zu seiner nationalen Selbstbehauptung // Die Bayern sind heute derjenige Stamm, der mit besonderem Eifer den Gedanken der nationalen Erneuerung erfaßt hat // wofern es gelingt, das deutsche Volk [...] durch Anteilnahme an seinen nationalen Geschicken zu politisieren; (präd. Vorkommen:) dann wird die deutsche Demokratie* national werden // daß der Klassenkampfgedanke nicht nur "der Form nach national" ist; (adverbial): Das Proletariat ist vorbestimmt, den sozialen Kampf gleichzeitig national zu führen; [...].

Während Konservativismus und konservativ Ausdrücke für die wahre Erkenntnis und entsprechend auch die wahre Vertretung nationaler Interessen sind, bilden Liberalismus und liberal die geradezu feindlichen Gegenwörter dazu. Sind mit diesen beiden Wortbildungsfeldern die Antipoden gekennzeichnet, so werden die Zwischenstufen durch Ausdrücke wie sozial oder demokratisch gekennzeichnet.

Deutschland um Deutschlands willen erhalten. Und er weiß, was er will. Der Nationalismus sagt nicht, wie der Patriotismus dies tut, daß das Deutsche erhaltenswert sei, weil es deutsch ist. Die Nation ist fur den Nationalisten kein Selbstzweck der von der Vergangenheit her klar und sichtbar und bereits erfullt vor uns liegt. Der Nationalismus ist durchaus auf die Zukunft der Nation gerichtet. Er ist konservativ, weil er weiß, da $\beta$ es keine Zukunft ohne Wurzelung in der Vergangenheit gibt. Und er ist politisch, weil er weiß, daß er der Vergangenheit wie der Zukunft nur sicher. sein kann, wofern er die Nation in der Gegenwart sichert." 
Je nationaler der Sozialismus ${ }^{22}$ bzw. die Demokratie, ${ }^{23}$ desto akzeptabler sind sie auch für Moeller van den Bruck. Je internationaler, desto mehr nähern sie sich dem nationalen Volksfeind Liberalismus an bzw. desto mehr sind diese an sich neutral bis positiv gekennzeichneten Ausdrücke vom liberalen Denken bereits zersetzt. Anders ausgedrückt: Moeller van den Bruck kann sich sowohl einen nationalen Sozialismus vorstellen als auch eine nationale Demokratie. Eine nationale Demokratie kann aber für ihn nur diejenige Demokratie sein, die dem geschichtlichen Dasein der deutschen Nation angemessen ist, und das heißt eine konservative, autoritäre Demokratie.

Ich würde an dieser Stelle die Vermutung wagen, dass Moeller mit der Präsidialregierung Brünings und von Papens, einem Hindenburg als Reichspräsidenten durchaus zufrieden gewesen wäre. Über seine Stellung gegenüber Hitler kann man nur spekulieren. Worüber man allerdings nach dem Lesen des Buches Gewissheit bekommt, ist, wen Moeller mit der Charakterisierung ,liberal ${ }^{\text {* }}$ als national unzuverlässig kennzeichnet. Es sind dieselben Personen und Gruppierungen, die von Konservativen wie Nationalsozialisten für den Dolchstoß verantwortlich gemacht werden, die von Hitler als Novemberverbrecher diffamiert und die von ihm im wirklichen dritten Reich verfolgt und ermordet werden. Es sind die Juden, ${ }^{24}$ die Freimaurer, ${ }^{25}$ die Sozialdemokraten und die Kommunisten. Weil er ihnen die Schuld am moralischen, intellektuellen und staatlichen Untergang der Nation zuschreibt, fordert auch Moeller ihre Vernichtung:

„Er [der konservative Mensch] weiß nur, daß die Welt immer wieder wird, was sie von Natur ist: konservativ. Und er trägt die Verantwortung dafür, daß sie auch in ihren Umwandlungen, die von der gleichen Natur aus nur krisenhaft, katastrophisch, ganz und gar unpazifistische sein können, gestützt, gebunden und zusammengefaßt wird, politisch vom Staate aus, und moralisch von den Menschen aus. Aber er weiß auch, daß die Welt, die bis zu dem Augenblicke konservativ gewesen ist, in dem der liberale Mensch in sie eintrat, erst dann wieder konservativ werden kann, wenn der liberale Mensch ausgetilgt worden ist." ${ }^{\text {"26 }}$

22 Vgl. Moeller van den Bruck 1923, 90f.: „Jedes Volk hat seinen eigenen Sozialismus.“ Oder 94f: „Wir sprechen schon heute von einem deutschen Sozialismus. [...] Wir verstehen unter deutschem Sozialismus vielmehr eine körperschaftliche Auffassung von Staat und Wirtschaft, die vielleicht revolutionär durchgesetzt werden muß, aber alsdann konservativ gebunden sein wird." Oder 144: „Der deutsche Sozialismus ist durch Liberalismus von Grund auf verdorben, und erst recht ist es der außerdeutsche".

23 Vgl. Moeller van den Bruck 1923, 171: „Die Frage der Demokratie ist nicht die Frage der Republik. In der Tat hängt das Schicksal der deutschen Demokratie von den Erfahrungen ab, die das deutsche Volk mit ihr machen wird. Eine Revolution hat niemals die Tendenz, revolutionär zu bleiben. Eine Revolution hat immer die Tendenz, konservativ zu werden. Wenn die Vermittlung der politischen Begriffe nie so unendlich wăre, dann würde die Möglichkeit einer konservativen Demokratie zu unseren selbstverständlichen Vorstellungen gehören. [...] Aber die deutsche Demokratie, die sich in Weimar ihre Verfassung gab, begreift erst sehr allmăhlich, daß sie ein überdauerndes Daseinsrecht nur dann besitzt, wenn sie nicht der Gegensatz der Monarchie ist, sondern deren Fortsetzung."; vgl. auch 151.

24. Moeller van den Bruck 1923, 24; 193.

25 Moeller van den Bruck 1923, $110 \mathrm{ff}$.

26 Moeller van den Bruck 1923, 324. 


\section{Literatur}

Adorno, Theodor W. u.a. $\left(1964^{3}\right)$ : The Authoritarian Personality. 2 Bde. New York.

Adorno, Theodor W. $\left(1996^{2}\right)$ : Studien zum autoritären Charakter. Frankfurt/M.

Albertin, Lothar (1972): Liberalismus und Demokratie am Anfang der Weimarer Republik. Düsseldorf.

Bohleber, Werner/Drews, Jörg (Hgg.) (1991): „Gift, das du unbewußt eintrinkst ..... Der Nationalsozialismus und die deutsche Sprache. Bielefeld.

Brunner, Otto/Conze, Werner/Koselleck, Reinhart (Hgg.) (1972ff.): Geschichtliche Grundbegriffe (GG). Historisches Lexikon zur politisch-sozialen Sprache in Deutschland. 9 Bde. Stuttgart.

Bussche, Raimund von dem (1998): Konservativismus in der Weimarer Republik. Die Politisierung des Unpolitischen. Heidelberg.

Bußmann, Walter (1960): Politische Ideologien zwischen Monarchie und Weimarer Republik. In: HZ 190, 55-77.

Conze, Werner (1972): „Demokratie“. In: Brunner/Conze/Koselleck 1972ff., Bd. 1,898 .

Dieckmann, Walther (1969): Sprache in der Politik. Heidelberg.

Dieckmann, Walther (1981): Politische Sprache. Heidelberg.

Ehlich, Konrad (Hg.) (1989): Sprache im Faschismus. Frankfurt/M.

Gerstenberger, Heide (1969): Der revolutionäre Konservatismus. Ein Beitrag zur Analyse des Liberalismus. Berlin.

Hermanns, Fritz (1994): Schlüssel-, Schlag- und Fahnenwörter: Zu Begrifflichkeit und Theorie der lexikalischen ,politischen Semantik“. Mannheim.

Hermanns, Fritz (1995): Sprachgeschichte als Mentalitätsgeschichte. Überlegungen zu Sinn und Form und Gegenstand historischer Semantik. In: Gardt, Andreas/Mattheier, Klaus/Reichmann, Oskar (Hgg.) (1995): Sprachgeschichte des Neuhochdeutschen. Gegenstände, Methoden, Theorien. Tübingen, 69-101.

Liedtke, Frank (Hg.) (1991): Begriffe besetzen: Strategien des Sprachgebrauchs in der Politik. Opladen.

Lobenstein-Reichmann, Anja (1998): Freiheit bei Martin Luther. Berlin.

Lobenstein-Reichmann, Anja (2000): Dichtung und Wahrheit in der Geschichtsschreibung. Lexikographie im Dienste historischer Erkenntnisfindung, dargestellt am Beispiel Luthers. In: Wiegand, Herbert Ernst (Hg.): Wörterbücher in der Diskussion IV. Tübingen, 175-202.

Lobenstein-Reichmann, Anja (im Druck): Die Dolchstoßlegende. Zur Konstruktion eines sprachlichen Mythos. Erscheint in: Muttersprache.

Lucács, Georg (1955): Die Zerstörung der Vernunft. Berlin.

Lucács, Georg (1966): Von Nietzsche bis Hitler oder Der Irrationalismus in der deutschen Politik. Frankfurt/M. 
Maas, Utz (1984): „Als der Geist der Gemeinschaft eine Sprache fand“. Sprache im Nationalsozialismus. Versuch einer historischen Argumentationsanalyse. Opladen.

Mann, Thomas (1918/19192): Betrachtungen eines Unpolitischen. Berlin.

Marcuse, Herbert. (1965): Der Kampf gegen den Liberalismus in der deutschen Staatsauffassung. In: Ders. Kultur und Gesellschaft I. Frankfurt/M., 17-55.

Moeller van den Bruck, Arthur (1923): Das Dritte Reich. Berlin.

Mohler, Armin $\left(1972^{2}\right)$ : Die konservative Revolution in Deutschland 19181932. Grundriß ihrer Weltanschauung. Ein Handbuch. Darmstadt.

Neurohr, Jean F. (1957): Der Mythos vom Dritten Reich. Zur Geistesgeschichte des Nationalsozialismus. Stuttgart.

Rödel, Helmut (1939): Moeller van den Bruck. Standort u. Wertung. Berlin [zugl. Diss. Leipzig.].

Schirmer, Dietmar (1992): Mythos-Heilshoffnung-Modernität. Politischkulturelle Deutungscodes in der Weimarer Republik. Opladen.

Schwierskott, Hans-Joachim (1962): Arthur Moeller van den Bruck und der revolutionäre Nationalismus der Weimarer Republik. Göttingen.

Sontheimer, Kurt (1971a): Deutschland zwischen Demokratie und Antidemokratie. Studien zum politischen Bewußtsein der Deutschen. München.

Sontheimer, Kurt (1971b): Thomas Mann und die Deutschen. München.

Sontheimer, Kurt $\left(1994^{4}\right)$ : Antidemokratisches Denken in der Weimarer Republik. München.

Stötzel, Georg/Wengeler, Martin (1995): Kontroverse Begriffe: Geschichte des öffentlichen Sprachgebrauchs in der Bundesrepublik Deutschland. Berlin.

Straßner, Erich (1987): Ideologie - Sprache - Politik. Grundfragen ihre Zusammenhangs. Tübingen.

Troeltsch, Ernst (1924): Spektator-Briefe. Aufsätze über die deutsche Revolution und die Weltpolitik 1918/22. Tübingen.

Troeltsch, Ernst (1925): Deutscher Geist und Westeuropa. Tübingen. 